\title{
Endourological Management of Forgotten Encrusted Ureteral Stents
}

\author{
Kusuma V. R. Murthy, S. Jayaram Reddy, D. V. Prasad
}

Department of Urology, Osmania General Hospital, Hyderabad, Andhra Pradesh, India

\begin{abstract}
Purpose: To present our experience and discuss the various endourological approaches for treating forgotten encrusted ureteral stents associated with stone formation.

Materials and Methods: From July 2006 to December 2008, 14 patients (11 men and 3 women) with encrusted ureteral stents were analyzed. The average indwelling time of the stent was 4.9 years (range 1 to 12). Plain-film radiography was used to evaluate encrustation, stone burden, and fragmentation of the stents. Intravenous urogram and a Tc99m diethylene triamine penta acetic-acid renogram was used to assess renal function.

Results: In seven patients, the entire stent was encrusted, in three patients the encrustation was confined to the ureteral and lower coil part of the stent, two patients had encrustation of the lower coil, and minimal encrustation was observed in two patients. Percutaneous nephrolithotomy was performed in 5 cases and retrograde ureteroscopy with intra-corporeal lithotripsy in 9 patients. Cystolithotripsy was used to manage the distal coil of the encrusted stent in eight patients. Simple cystoscopic removal of the stents with minimal encrustation was carried-out in two cases. Looposcopy and removal of the stent was performed in one patient with an ileal conduit and retained stent. Only one patient required open surgical removal of the stent. Thirteen out of 14 patients were rendered stone and stent free in one session. All except two stents were removed intact and stone analysis of encrustation and calcification revealed calcium oxalate and calcium phosphate in the majority of the cases.

Conclusion: Endourological management of forgotten encrusted stents is highly successful and often avoids the need for open surgical techniques.
\end{abstract}

Key words: stents; ureteral; forgotten; lithiasis; management; endourology

Int Braz J Urol. 2010; 36: 420-9

\section{INTRODUCTION}

Ureteral stents are widely used in urological practice. They are mainly indicated after any ureteral surgery and for managing ureteral obstruction due to intrinsic or extrinsic causes like stones, strictures, uretero-pelvic junction obstruction, retroperitoneal fibrosis, malignancies, and congenital anomalies (15). They are also placed after iatrogenic injuries to the ureter and before any complex abdominal procedure for identification and protection of the ureters (6). Because of their wide spread usage, complications due to these stents have also increased like, stent encrustation, stent fragmentation, stone formation and recurrent urinary tract infection $(7,8)$. Retention of ureteral stents, often due to poor compliance of the patient is not uncommonly seen (9). If left untreated, these retained stents result in significant morbidity and mortality. Various methods of treatment combinations of extracorporeal shock wave lithotripsy 
(SWL), cystolithotripsy (CLT) retrograde ureteroscopy with intracorporeal lithotripsy, percutaneous nephrolithotomy (PCNL) and open surgery have been used for retrieval of these encrusted stents (10-14). We present our experience with the management of these forgotten stents, associated with significant encrustation and stone burden in 14 patients.

\section{MATERIALS AND METHODS}

Fourteen patients (11 men and three women) with forgotten ureteral stents with severe encrustation, who presented at our department between July 2006 and December 2008, were treated. Information was obtained through a retrospective review of patients records. The mean patient age was 42.4 years (range 27-55 years) and the average indwelling time of the stent was 4.9 years (range 1-12 years).All the stents were placed elsewhere. Poor compliance and inability of the treating surgeon to counsel the patients were the reasons for retention of these stents. All the patients were evaluated for stent encrustation and associated stone burden by plain-film radiography and intravenous urogram. In patients with non visualized kidneys on intravenous urogram, Tc99m diethylene triamine penta acetic-acid (DTPA) renogram was done to estimate the renal function. Treatment decision was made on clinical and radiological findings. Before intervention, all patients had negative urine cultures, and antibiotic prophylaxis was given for all cases.

Combined endourological procedures PCNL, Cystolithotripsy (CLT), retrograde ureteroscopy with intracorporeal lithotripsy were performed in one session. Retrograde ureteroscopy was performed using $8 / 9.8 \mathrm{~F}$ and $6 / 7.5 \mathrm{~F}$ semi rigid ureteroscope, under fluoroscopic guidance. Intracorporeal lithotripsy was performed with a pneumatic lithotripter. PCNL was carried-out using a rigid 24F nephroscope. In stents with minimal encrustation on plain-film radiography, a gentle attempt is made for removal with the help of grasping forceps passed through the cystoscope under local anesthesia and fluoroscopic guidance. For patients with encrustation and stone burden involving the lower coil, ureteric (body) or whole of the stent, initially, cystolithotripsy, retrograde ureteroscopy and intra corporeal lithotripsy was performed in the dorsal lithotomy position. Following this, a gentle attempt was made to retrieve the stent with the help of an ureteroscopic grasper. If the stent failed to uncoil, a ureteric catheter was placed adjacent to the encrusted stents for injection of radio-contrast material to delineate the renal pelvis and the calyces. Then the patient was placed in the prone position and PCNL of the upper coil of the encrusted stent along with calculus was done. The approach to the collecting system was through the lower calyx and middle posterior calyx and no patient required upper pole or supra costal access. A 14F nephrostomy tube was kept indwelling for 48 hours, in patients who required PCNL. Stone analysis and encrustation analysis was done in all cases. Post operatively, plain-film radiography was done to confirm the stone free and stent free status.

\section{RESULTS}

The patient characteristics, initial indications for stenting, indwelling time, site of encrustation, type of procedure performed and length of hospital stay are shown in Table-1. The entire stent was encrusted in 7 patients; three patients had predominantly ureteric (body) and lower coil encrustation (Figure-1) and in 2 patients, there was minimal encrustation. In 11 out of 14 patients, the initial indication for stent placement was for urinary stone disease. General anesthesia was required for removal of the encrusted stents in eleven patients, and three patients were managed as outpatients under local anesthesia. The mean hospital stay in 11 patients was 4.9 days. As shown in Table-1, CLT was required to treat the distal end of the stent in seven cases. PCNL was done in 5 cases. Retrograde ureteroscopy and intra corporeal lithotripsy was performed in eight cases. Simple cystoscopic removal of the stent under fluoroscopic guidance was done in two cases.

A 54-year-old male patient who underwent radical cystectomy and ileal conduit 4 years previously, presented with left loin pain and dysuria for 3 months. Plain-film radiography of the abdomen revealed a retained stent on the left side with minimal encrustation. Looposcopy with 24F nephroscope and removal of the retained stent was done with the help of a grasping forceps under fluoroscopic guidance. 


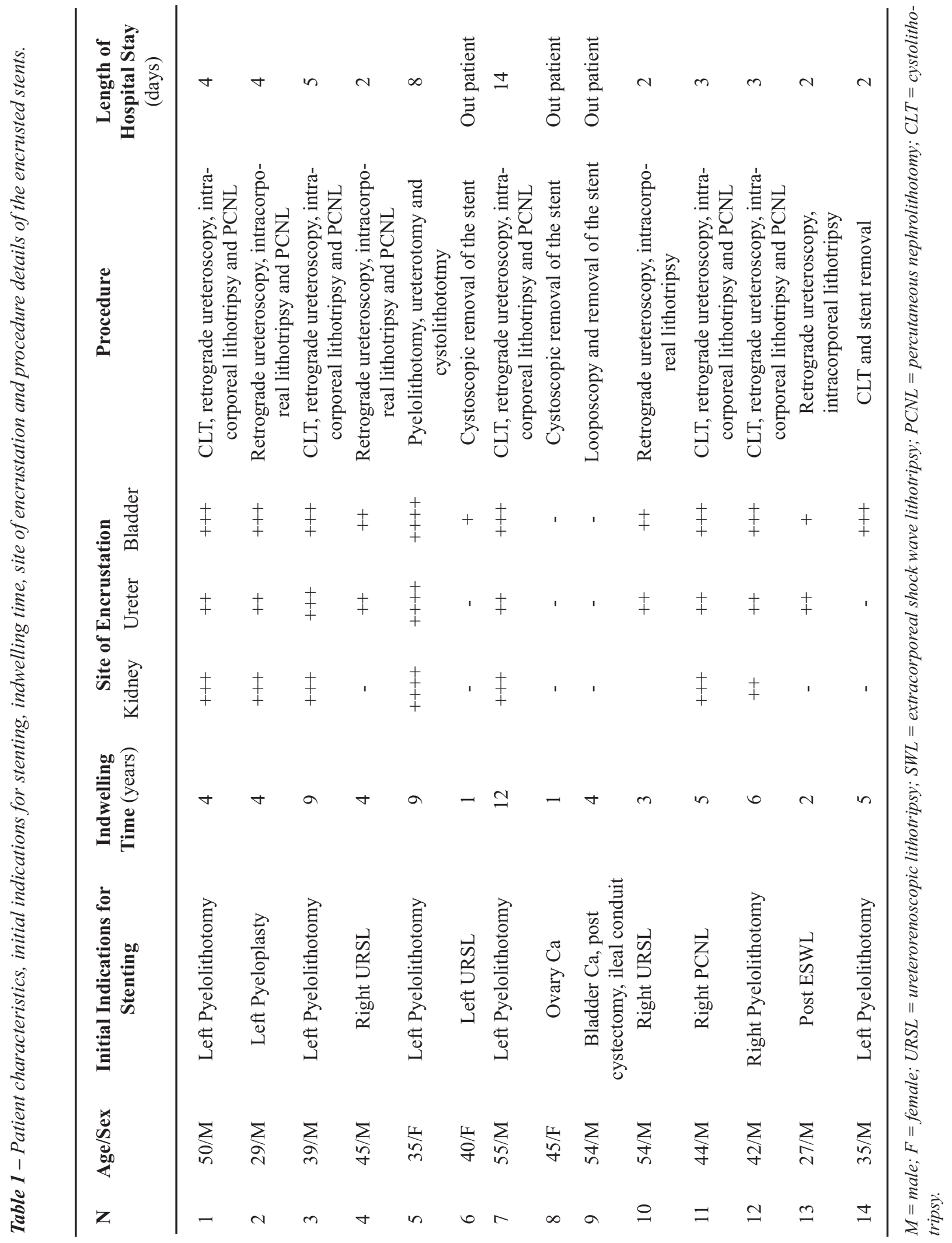




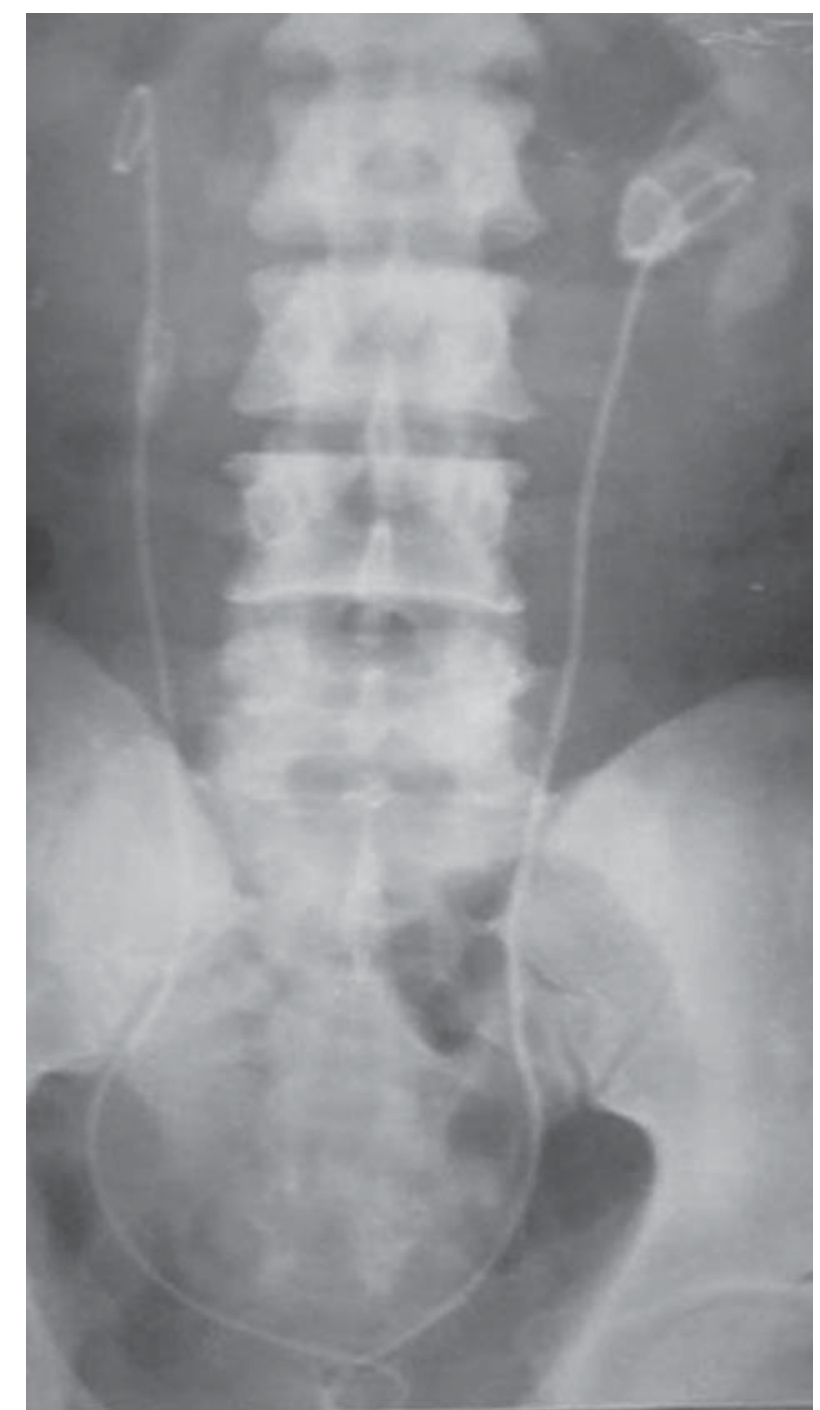

Figure 1 - Plain-film radiography of a patient who presented with renal failure. Left side retained ureteral stent with extensive calcification at the proximal end of the stent. On the right side, a ureteric calculus was seen and a stent was placed preoperatively to normalize serum creatinine level.

One patient presented with a left retained ureteral stent for 9 years and right upper ureteric calculus, and a serum creatinine level of $5.4 \mathrm{mg} \%$. Initially, a right ureteral stent was placed to normalize the renal parameters, and subsequently, a push back PCNL was done for the right ureteric calculus to achieve complete stone clearance. On the left side, a retrograde ureteroscopy with intracorporeal lithotripsy and PCNL was required to fragment and retrieve the encrusted stent (Figure-2).
Another patient presented with retained stent for 9 years following pyelolithotomy on the left kidney. Plain-film radiography showed extensive calcification of the entire stent. Intravenous urogram and DTPA renogram showed good renal function. CLT of the lower coil of the encrusted stent was attempted, but the pneumatic lithotripter failed to fragment the stone. Since we do not have the facility of laser lithotripsy open surgical removal by cystolithotomy, ureterotomy and pyelolithotomy was done to retrieve the stent (Figure-3).

Percutaneous nephrostomy was carried-out in one patient who presented with acute pyelonephritis. The dwelling time of the encrusted stent in this patient was 12 years. After negative bacterial culture of the blood and urine, the patient underwent surgery. CLT, retrograde ureteroscopy with intracorporeal lithotripsy was required for removal of the stent. On the second postoperative day, he developed urosepsis, requiring broad spectrum antibiotics and intensive care management. This patient had a prolonged hospital stay of 14 days. Thirteen out of 14 patients $(93 \%)$ were rendered stone free and stent free after a single anesthetic session. Re-stenting was done in one patient requiring open surgery and subsequently, his stent was removed after four weeks. No intra operative complications occurred in any patient. All the stents were removed intact except in two patients, who had fragmented stents at presentation. Stone analysis showed calcium oxalate and phosphate in the majority of cases. Struvite stones were seen in two cases.

\section{COMMENTS}

Forgotten ureteral stents are observed in urologic practice because of poor compliance of the patient or failure of the physician to adequately counsel the patient. These forgotten stents can produce considerable morbidity and mortality, due to extensive encrustation with significant stone burden, knot formation, upward migration and fragmentation $(7,15)$. Encrustation of forgotten stents associated with large stone burden is a serious problem, due to complications like recurrent urinary tract infection, hematuria, obstruction and renal failure (16). The deposition of encrusted material on retained ureteral 

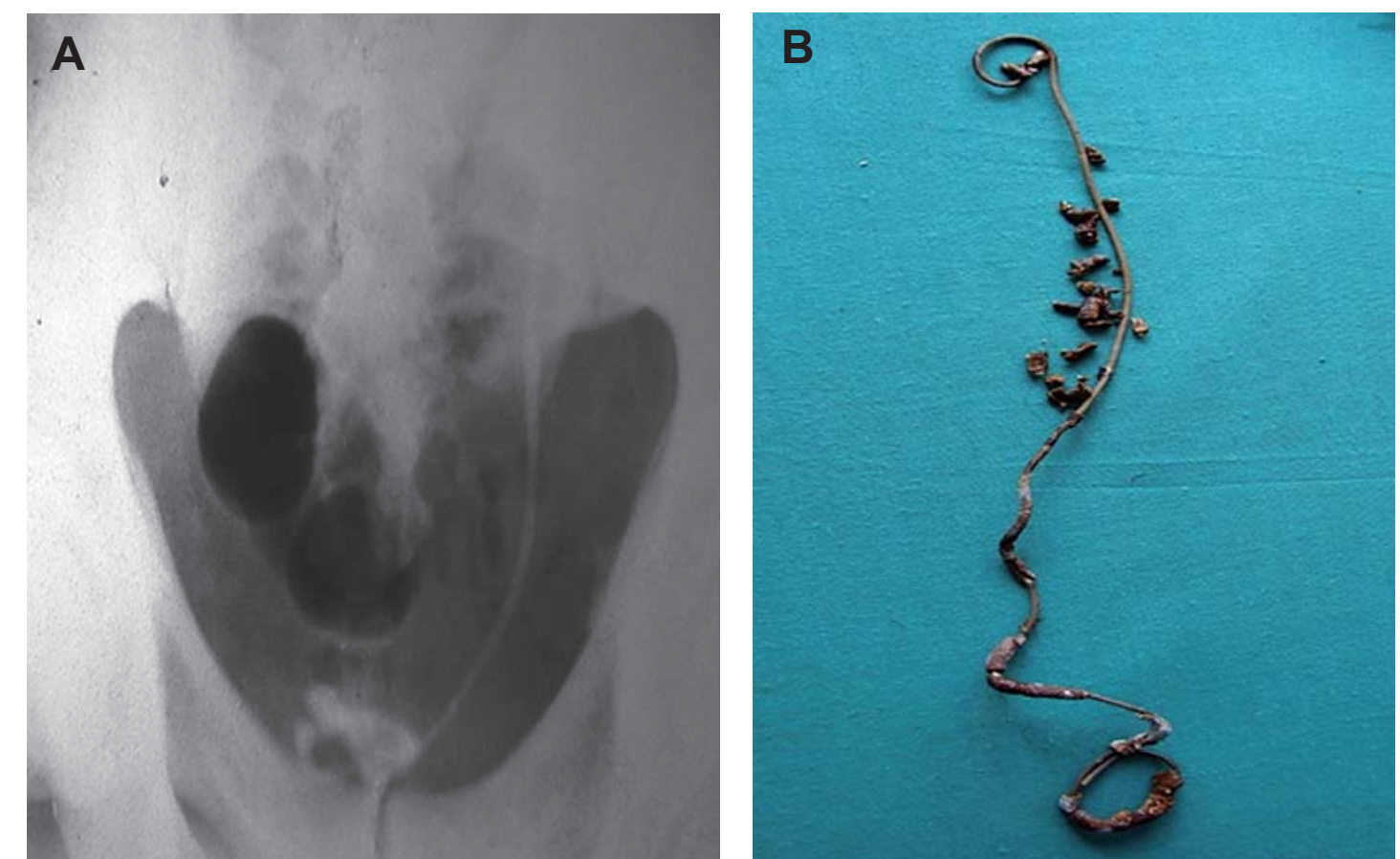

Figure 2-A) Plain-film radiography of a 54 yr old male showing encrusted stent with calculus formation at the lower part and bladder coil of the stent. B) Retrieved stent showing extensive encrustation and stone formation.

stents can occur in both infected and sterile urine. The mechanism of encrustation in infected urine is a result of organic components in the urine crystallizing out onto the surface of biomaterial and becoming incorporated into a bacterial biofilm layer. Urease produced by the adhered bacteria hydrolyses the urea to produce ammonia. This elevates urinary $\mathrm{pH}$, favoring the precipitation of magnesium and calcium as struvite and hydroxyl apatite $(17,18)$. Although the exact mechanism of encrustation in sterile urine is unclear, it appears to be dependent on the $\mathrm{pH}$, ionic strength and biomaterial hydrophobic properties (19). The degree of encrustation is dependant on the dwelling time. El faqih et al. found that encrustation increased from $9.2 \%$ at $<6$ weeks to $47.5 \%$ at six to 12 weeks to $76.3 \%$ at $>12$ weeks of dwelling time (20). Other factors implicated in the increased incidence of encrustations are chronic recurrent stone formers, metabolic predisposition to stone disease, congenital renal anomalies, malignant urinary obstruction and pregnancy (21).

Fragmentation is another important complication of the forgotten stents. It is the result of loss

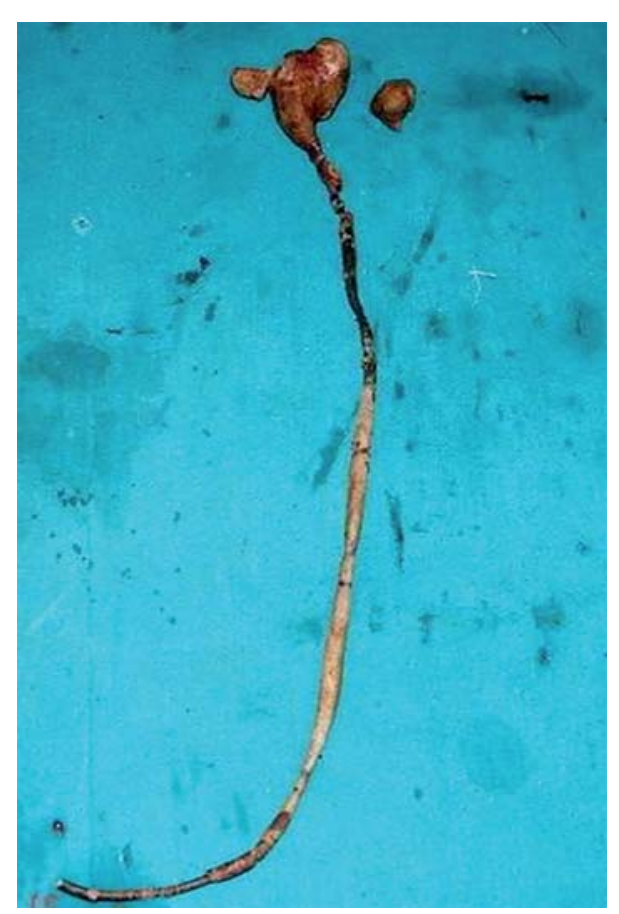

Figure 3-Retrieved stent with extensive calcification. This patient had a retained stent for 9 years. Open surgery was required to remove the stent. 
of tensile strength, which is due to hardening and degeneration of the stent polymers (22). The risk of encrustation and fragmentation is dependant on the type of material of the stent. Silicone was found to be least prone to encrustation, followed by polyurethane, silitek, percuflex and hydro gel coated polyurethane (23). Fragmentations of polyurethane stents are four times as frequent as the silicone stents (9). In our series, fragmentation of the lower coil of the stent is seen in two cases at the time of presentation. The indwelling time in both the cases was five years. All the retrieved encrusted stents in our series were made of polyurethane.

Retained ureteral stents with encrustation is a challenging problem for endourologists. Very often, multiple endourological approaches are needed because of encrustations and the associated stone burden that may involve bladder, ureter and kidney. This may require single or multiple endourological sessions or rarely open surgical removal of the encrusted stents. Singh et al. described multiple accesses and approaches including open surgery to treat the retained stents (24). Borboroglu et al. also reported the endourological treatment of four patients with severely encrusted ureteral stents with a large stone burden. All patients required two to six endourological approaches (average 4.2) performed at one or multiple sessions, to achieve stone-free and stent-free status. These authors concluded that percutaneous nephrolithotomy and ureteroscopy are often necessary for treating a severely encrusted stent and associated stone burden (13). One stage removal of 12 encrusted retained ureteral stents has been reported by Bukkapatnam et al., in ten patients. Of these, 11 were managed by ureteroscopy alone and in one patient; the stone was treated through a percutaneous approach. They concluded that, these stents can be removed in one sitting with minimal morbidity and short hospital stay (25). Using a combination of SWL, PCNL, CLT, ureteroscopy with intra corporeal lithotripsy, clearance rates ranging from 75 to $100 \%$ have been reported $(10,12,22)$.

The site of encrustation, associated stone burden and the function of the affected kidney often dictate the method of access and treatment (Figure-4). Our approach towards management of these difficult stents is based on the findings on plain-film radiog- raphy. The proximal, distal coils and the body of the stent are examined for encrustation, calcification and fragmentation. We did not find any additional benefit in the management plan with the use of non contrast computed tomography. Intravenous urogram and DTPA renogram is obtained to determine the function of the kidney. Nephrectomy is done for non salvageable function of the kidney. Nephrostomy or placement of second stent is done, if the patient presented with pyelonephritis and sepsis. It is possible to put a second stent adjacent to the encrusted stent because the ureter is dilated in majority of these cases.

Extracorporeal shock wave lithotripsy (SWL) is the initial treatment with stents with minimal encrustation. However, in our series, no patient required SWL because of extensive stone burden in majority of cases. If there are no encrustations visible on plainfilm radiography, our approach is cystoscopic removal using a grasping forceps under local anesthesia with fluoroscopic guidance. Gentle traction on the stent is applied, if patient complains of pain and if the stent does not uncoil, the procedure is abandoned. An important precaution during the procedure is to avoid using excessive force, which can result in breakage of the stent along with ureteral injury or ureteral avulsion.

The next stage is CLT with the help of pneumatic lithotripter on stents with minimal encrustation and those with lower coil encrustation. This followed by gentle pull under fluoroscopic guidance. If the cystoscopic approach fails, and in patients with encrustation involving the ureteric portion of the stent, the next approach is under anesthesia, a safety guide wire is passed along the retained stent and ureteroscope is passed retrograde. Calcifications over the stent can be fragmented with a pneumatic lithotripter or laser energy, while carefully advancing the ureteroscope into the renal pelvis. After all the encrustations and calcification have been fragmented, the stent is gently removed with the help of grasping forceps passed through the ureteroscope under fluoroscopic guidance. Following removal of the stent, it is mandatory to do a retrograde uretrogram and check ureteroscopy to rule out a ureteric injury. If any signs of ureteric injury or contrast extravasation present, the patient should be re-stented.

For stents with large stone burden and those stents which fail to be retrieved by the above-men- 


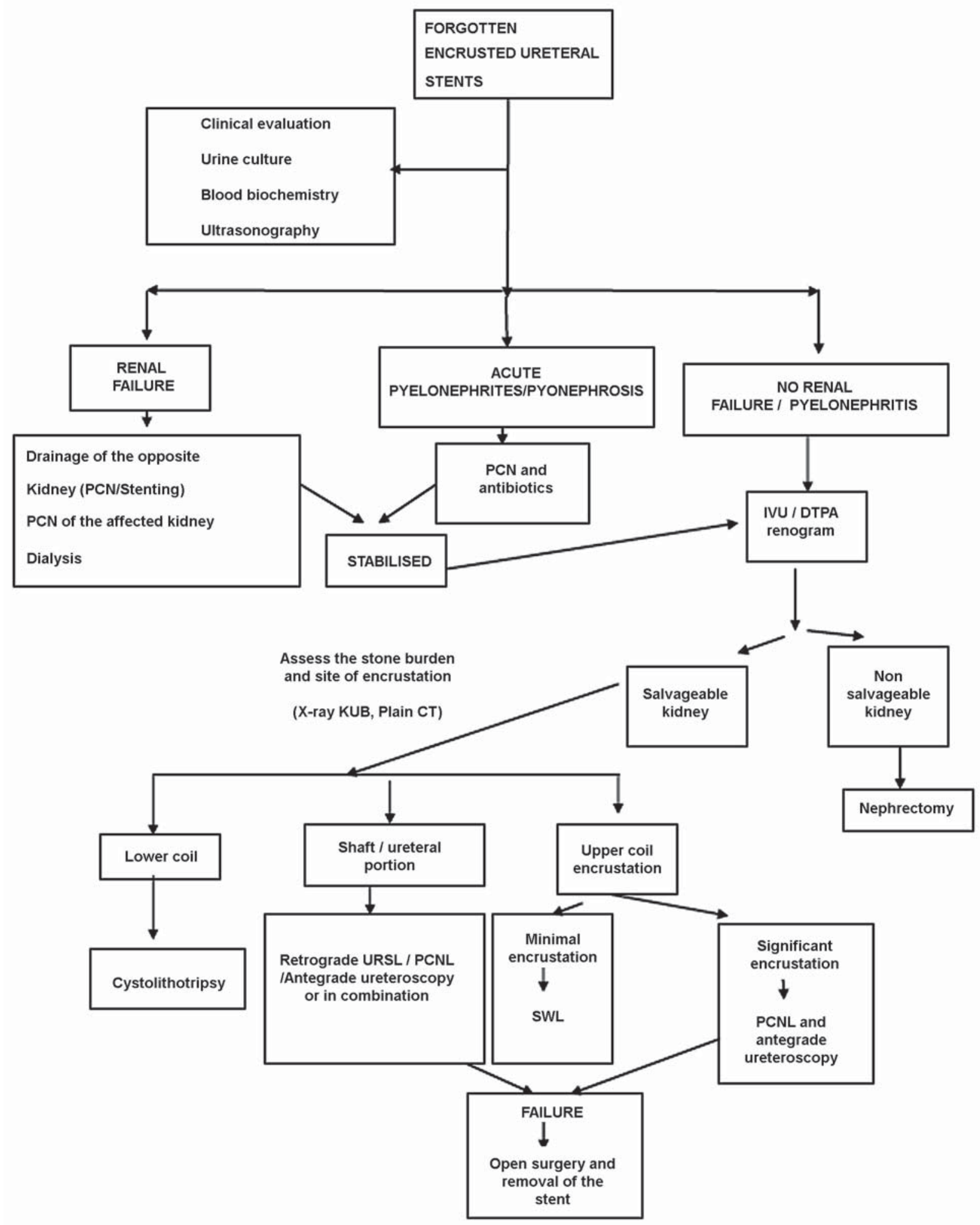

Figure 4 - Algorithm for the management of forgotten encrusted stents. CT = computer tomography; DTPA = diethylenetriamine penta-acetic acid; $S W L=$ extracorporeal shock wave lithotripsy; IVU = Intravenous Urogram; KUB = kidneys, ureters, and bladder; $P C N L=$ percutaneous nephrolithotomy; URSL = ureterorenoscopic lithotripsy. 
tioned techniques, a 5F ureteric catheter is placed to enable the injection of radio contrast material into the renal pelvis and calyces as an aid to subsequent percutaneous access and the patient is placed in the prone position. Percutaneous access is established by a lower calyceal or middle calyceal puncture and the proximal coil of the stent along with stone is fragmented. The stent is gently removed under fluoroscopic guidance through the percutaneous nephrostomy tract.

Using the above-mentioned approach, it was possible to remove all stents in 13 out of 14 patients, using the endourological approach alone under a single anesthesia. Open surgery was done in one case because of the extensive stone burden and failure of the pneumatic lithotripter to fragment the stone. Based on our method of approach, an algorithm has been proposed for the management of these stents (Figure-4).

Although, endourological management of these stents achieves success in majority of the cases with minimal complications, the best treatment that remains is prevention of this complication. The treating physician should be very selective in placing the stents and they must be tracked very closely by documenting insertion and removal of the stents. All patients should be counseled with respect to the complications of long term use and advised when their stent should be changed. As mentioned earlier, the degree of encrustation is dependant on the indwelling time, so, it is necessary to keep the indwelling time to as short as possible. Various authors have reported that indwelling time between 2-4 months is safe $(9-12,20)$. For patients requiring stents beyond this period, they should be kept on prophylactic antibiotics and have their stents frequently changed.

It is interesting to note that, two of three patients who did not have stents for stone disease, were able to have their stents simply removed by cystoscopy, while this was successful in none of the stone formers. The reason for this could be due to increase risk of encrustation and stone formation in patients who have a history of stone disease. This underscores the importance of frequent monitoring in these groups of patients to avoid life threatening complications.

It is also important to maintain a proper record of all stents inserted and keep a track of their due date of removal. Some authors have proposed a computerized tracking program for removal stents (26). Coatings such as hydrophilic polymers, heparin, pentosan polysulfate, or oxalate -degrading enzymes have been used in an attempt to reduce encrustation (27-30). The use of bio-degradable compound of poly-L-lactic and glycolic acids which are designed to disintegrate can eliminate the problem of retention and encrustation of the stents in the near future (31).

\section{CONCLUSION}

Encrustation and stone formation in forgotten stents often lead to life threatening complications and pose a challenging management task for the treating surgeon.

The use of various combinations of endourological techniques can achieve effective stent and stone treatment after a single anesthesia session with minimal morbidity and short hospital stay. Imaging and assessment of the degree of stone burden is important, before making any attempt to remove these stents. Maintenance of efficient log book under direct supervision of treating surgeon and proper patient counseling is required to prevent this complication.

\section{ACKNOWLEDGEMENTS}

Drs. Panduranga Rao, T. Jagadeshwar, Srinivas, Purusotham, Sudershan, and Roopali provided support to this study.

\section{CONFLICT OF INTEREST}

None declared.

\section{REFERENCES}

1. Saltzman B: Ureteral stents. Indications, variations, and complications. Urol Clin North Am. 1988; 15: 481-91. 


\section{Management of Forgotten Ureteral Stents}

2. Chew BH, Knudsen BE, Denstedt JD: The use of stents in contemporary urology. Curr Opin Urol. 2004; 14: 111-5.

3. Hepperlen TW, Mardis HK, Kammandel H: The pigtail ureteral stent in the cancer patient. J Urol. 1979; 121: $17-8$.

4. Gogas J, Markopoulos C, Kouskos E, Gogas H, Kiriakou V: Metastatic retroperitoneal and mediastinal fibrosis as first sign of recurrence of breast cancer. Eur J Surg. 2001; 167: 715-8.

5. Park DS, Park JH, Lee YT: Percutaneous nephrostomy versus indwelling ureteral stents in patients with bilateral nongenitourinary malignant extrinsic obstruction. J Endourol. 2002; 16: 153-4.

6. Kuno K, Menzin A, Kauder HH, Sison C, Gal D: Prophylactic ureteral catheterization in gynecologic surgery. Urology. 1998; 52: 1004-8.

7. Damiano R, Oliva A, Esposito C, De Sio M, Autorino R, D'Armiento M: Early and late complications of double pigtail ureteral stent. Urol Int. 2002; 69: 13640 .

8. Schulze KA, Wettlaufer JN, Oldani G: Encrustation and stone formation: complication of indwelling ureteral stents. Urology. 1985; 25: 616-9.

9. Monga M, Klein E, Castañeda-Zúñiga WR, Thomas $\mathrm{R}$ : The forgotten indwelling ureteral stent: a urological dilemma. J Urol. 1995; 153: 1817-9.

10. Mohan-Pillai K, Keeley FX Jr, Moussa SA, Smith G, Tolley DA: Endourological management of severely encrusted ureteral stents. J Endourol. 1999; 13: 3779.

11. Flam TA, Brochard M, Zerbib M, Debre B, Steg A: Extracorporeal shock-wave lithotripsy to remove calcified ureteral stents. Urology. 1990; 36: 164-5.

12. Somers WJ: Management of forgotten or retained indwelling ureteral stents. Urology. 1996; 47: 4315.

13. Borboroglu PG, Kane CJ: Current management of severely encrusted ureteral stents with a large associated stone burden. J Urol. 2000; 164: 648-50.

14. Lam JS, Gupta M: Tips and tricks for the management of retained ureteral stents. J Endourol. 2002; 16: 73341.

15. Eisner B, Kim H, Sacco D: Repeat knot formation in a patient with an indwelling ureteral stent. Int Braz J Urol. 2006; 32: 308-9.

16. Singh V, Srinivastava A, Kapoor R, Kumar A: Can the complicated forgotten indwelling ureteric stents be lethal? Int Urol Nephrol. 2005; 37: 541-6.

17. Wollin TA, Tieszer C, Riddell JV, Denstedt JD, Reid G: Bacterial biofilm formation, encrustation, and antibiotic adsorption to ureteral stents indwelling in humans. J Endourol. 1998; 12: 101-11.

18. Robert M, Boularan AM, El Sandid M, Grasset D: Double-J ureteric stent encrustations: clinical study on crystal formation on polyurethane stents. Urol Int. 1997; 58: 100-4.

19. Keane PF, Bonner MC, Johnston SR, Zafar A, Gorman SP: Characterization of biofilm and encrustation on ureteric stents in vivo. Br J Urol. 1994; 73: 687-91.

20. el-Faqih SR, Shamsuddin AB, Chakrabarti A, Atassi R, Kardar AH, Osman MK, et al.: Polyurethane internal ureteral stents in treatment of stone patients: morbidity related to indwelling times. J Urol. 1991; 146: 148791.

21. Lojanapiwat B: Endourological management of severely encrusted ureteral stents. J Med Assoc Thai. 2005; 88: 1203-6.

22. Zisman A, Siegel YI, Siegmann A, Lindner A: Spontaneous ureteral stent fragmentation. J Urol. 1995; 153: 718-21.

23. Tunney MM, Keane PF, Jones DS, Gorman SP: Comparative assessment of ureteral stent biomaterial encrustation. Biomaterials. 1996; 17: 1541-6.

24. Singh I, Gupta NP, Hemal AK, Aron M, Seth A, Dogra PN: Severely encrusted polyurethane ureteral stents: management and analysis of potential risk factors. Urology. 2001; 58: 526-31.

25. Bukkapatnam R, Seigne J, Helal M: 1-step removal of encrusted retained ureteral stents. J Urol. 2003; 170: 1111-4.

26. Ather MH, Talati J, Biyabani R: Physician responsibility for removal of implants: the case for a computerized program for tracking overdue double-J stents. Tech Urol. 2000; 6: 189-92.

27. Gorman SP, Tunney MM, Keane PF, Van Bladel K, Bley B: Characterization and assessment of a novel poly(ethylene oxide)/polyurethane composite hydrogel (Aquavene) as a ureteral stent biomaterial. J Biomed Mater Res. 1998; 39: 642-9.

28. Riedl CR, Witkowski M, Plas E, Pflueger H: Heparin coating reduces encrustation of ureteral stents: a preliminary report. Int J Antimicrob Agents. 2002; 19: 507-10.

29. Watterson JD, Cadieux PA, Beiko DT, Cook AJ, Burton JP, Harbottle RR, et al.: Oxalate-degrading enzymes from Oxalobacter formigenes: a novel device coating to reduce urinary tract biomaterial-related encrustation. J Endourol. 2003; 17: 269-74.

30. Zupkas P, Parsons CL, Percival C, Monga M: Pentosanpolysulfate coating of silicone reduces encrustation. J Endourol. 2000; 14: 483-8. 
31. Lingeman JE, Preminger GM, Berger Y, Denstedt JD, Goldstone L, Segura JW, et al.: Use of a temporary ureteral drainage stent after uncomplicated ureteroscopy: results from a phase II clinical trial. J Urol. 2003; 169: 1682-8.

\section{Correspondence address:}

Dr. K. V. R. Murthy

Department of Urology

Osmania General Hospital

Afzal gunj, Hyderabad 500012

Andhra Pradesh, India

Fax: + 9140 2460-0260

E-mail: murthy.kusuma@rediffmail.com 